Linguistique, littérature, didactique

$177-178$ | 2018

Langage oral à l'école maternelle. Étude d'un corpus

homogène

\title{
Reformulation, re-construction : comprendre, entendre, s'approprier un texte complexe
}

Reformulation, re-construction: understanding, perceiving, appropriating a complex text

\section{Natacha Espinosa et Sarah de Vogüé}

\section{CpenEdition}

Journals

Édition électronique

URL : http://journals.openedition.org/pratiques/4332

DOI : $10.4000 /$ pratiques.4332

ISSN : 2425-2042

Éditeur

Centre de recherche sur les médiations (CREM)

Référence électronique

Natacha Espinosa et Sarah de Vogüé, «Reformulation, re-construction : comprendre, entendre, s'approprier un texte complexe », Pratiques [En ligne], 177-178 | 2018, mis en ligne le 20 juillet 2018, consulté le 19 avril 2019. URL : http://journals.openedition.org/pratiques/4332 ; DOI : 10.4000/ pratiques. 4332

Ce document a été généré automatiquement le 19 avril 2019

(ㄷ) Tous droits réservés 


\title{
Reformulation, re-construction : comprendre, entendre, s'approprier un texte complexe
}

\author{
Reformulation, re-construction: understanding, perceiving, appropriating a \\ complex text
}

Natacha Espinosa et Sarah de Vogüé

1 Le dispositif élaboré autour de la lecture du texte Danger dans le potager pose des questions importantes relatives à ce qui se joue dans les apprentissages du langage chez l'enfant, questions qui débordent largement le champ de la didactique et mobilisent tous les linguistes nourrissant un intérêt pour les productions langagières d'enfants en général, et à l'occasion d'un apprentissage à la maternelle en particulier. Notre parti ici est de considérer que le corpus qui nous est proposé permet d'avancer dans la compréhension des phénomènes langagiers se déroulant dans une activité de narration collective, mais aussi plus largement qu'il nous céclaire sur ce que signifie apprendre à parler chez l'enfant :

- Qu'est-ce que les enfants sont en train d'acquérir ? Quelles sont notamment, au niveau de la structuration du langage, les formes et les constructions qu'ils mobilisent pour structurer leur discours d'une part, reconstruire collectivement l'histoire d'autre part?

- Mais aussi, que recouvre cette maitrise du langage? Qu'est-ce que comprendre puis raconter un texte? Qu'est-ce que raconter ensemble un texte? Qu'est-ce que cela favorise dans le développement du langage de l'enfant?

\section{Cadrage théorique}

Le dispositif repose sur le fait de faire raconter, de faire reconstruire un texte, collectivement, par des élèves de maternelle. Cette reconstruction collective s'opère à partir des verbalisations des élèves, motivées après l'écoute de ce texte par les questionnements des enseignants. Pour reconstruire le texte, les élèves vont donc 
s'appuyer sur leur mémorisation des éléments entendus: éléments concernant sa construction, ses personnages, ses lieux... Il s'agit de remobiliser les images mentales qui se sont formées chez chacun d'eux au cours des écoutes du texte, celles-ci étant nourries à la fois par leur compréhension de l'histoire, par leur compréhension des relations entre les personnages et entre les actions et par leur expérience singulière du monde. Mais au cours de la reconstruction collective, chaque élève va s'appuyer aussi sur ce que les autres élèves proposent et disent de leur compréhension et de leur interprétation.

3 Pour faire cette étude, nous avons choisi d'analyser d'un point de vue linguistique la façon dont les élèves parlent du texte : à travers des reformulations, qui peuvent être des répétitions au sens strict, des paraphrases, des précisions ou des explicitations.

Dans le prolongement du cadre définitoire posé par C. Fuchs (1982) puis É.Gülich et T. Kotschi (1987), les paraphrases correspondent à une relation d'équivalence sémantique entre deux énoncés reposant sur des opérations linguistiques qui s'appuient sur différentes formes ou marqueurs linguistiques. Les reformulations peuvent cependant aussi apporter des précisions, soit qu'elles rajoutent des éléments, soit qu'elles modulent l'énoncé de départ, soit qu'elles visent à clarifier le contenu une première fois formulé (Le Bot, Schuwer \& Richard, 2008). Elles peuvent aussi avoir des fins argumentatives quand il s'agit de contrer ou de renchérir sur ce qui est repris (Steuckardt, 2009).

5 Les reformulations que l'on observe dans le corpus analysé s'appuient bien sur un invariant sémantique supposé avec l'énoncé source (le texte), mais aussi sur les propos des pairs, qui doivent être modifiés, précisés ou complétés pour arriver à re-construire un texte commun.

Des études sur le rôle des interactions enseignant-élève dans la construction des savoirs scolaires en général (par exemple Bautier, 2001 ; Garcia-Debanc, 2006 ; Jaubert, Rebière \& Pujo, 2010) s'appuient également sur la reformulation, celle-ci étant plus particulièrement observée du point de vue de l'enseignant, qui reformule ce que disent les élèves pour les aider à mieux dire, à mieux expliquer, mais aussi à corriger leurs propositions (Rabatel, 2010 ; Pégaz-Paquet, 2009). Dans notre étude, il s'agit d'observer et de rendre compte de quelques exemples de reformulation venant non pas des enseignants, mais des élèves eux-mêmes, qui, par ces procédés, donnent à voir ce qu'ils ont compris et retenu du texte, mais donnent à voir aussi comment ils construisent un discours à partir d'un texte écrit et entendu d'une part, à partir des verbalisations de leurs pairs d'autre part. L'étude se donne ainsi un double objectif qui est à la fois de mesurer ce que la reformulation leur permet de faire en termes de reconstruction et de restitution d'un texte, et de mesurer les compétences linguistiques qu'ils développent ce faisant pour construire un langage explicite et structuré - ce langage explicite et structuré étant nécessaire pour les préparer d'une certaine façon à aborder l'écrit (Lentin, 2009).

7 Autrement dit, les procédés de reformulation des élèves nous paraissent devoir être étudiés non seulement en relation avec le texte à restituer, mais aussi en relation avec la façon dont leur langage oral se structure, les deux objectifs se rejoignant dans l'apprentissage de ce qu'est raconter oralement un texte écrit, cela dans la perspective d'un accès ultérieur au lire-écrire. La reformulation, sous toutes ses formes rapidement évoquées ci-dessus, sera ici abordée comme un processus d'apprentissage du langage, un phénomène langagier qui agit dans la construction des savoirs sur le texte mais aussi dans le développement des compétences langagières (Volteau, 2015). 
Il nous semble en outre que la reformulation peut être tenue pour un élément essentiel de ces compétences langagières elles-mêmes. En ceci, on rejoint la perspective qui est celle d'A. Culioli (1999) quand il place reformulation et explicitation - qui constitue ce qu'il appelle l'activité épilinguistique - au cœur de la faculté de langage. De fait, la reformulation fait appel à un travail actif de construction verbale, qui s'appuie sur des compétences paraphrastiques effectives (Martinot, 2010) ${ }^{1}$. De ce point de vue, le dispositif proposé offre aux élèves l'occasion de s'entrainer à paraphraser, à reformuler, et donc à « jouer avec la diversité des formes d'expression » (Fuchs, 1994, p. 12) favorisant de cette manière leurs verbalisations, et « leur aisance langagière et cognitive ». Et en retour, le fait de reformuler collectivement des petits bouts du texte nous semble favoriser les relations qui permettent de construire des réseaux de significations et de compréhension qui vont alors avoir une incidence sur la représentation mentale du texte et sa reconstruction.

\section{Positionnement méthodologique}

Nous nous proposons d'étudier les ressorts linguistiques qui sont mobilisés par ces enfants non encore lecteurs pour arriver à restituer le texte entendu : non pas seulement les formes qu'ils utilisent, mais la façon même dont ils procèdent. Pour ce faire, nous avons opté pour une approche qualitative des données, avec un objectif d'abord descriptif, en nous centrant sur des extraits qui nous paraissent particulièrement éclairants.

Dans une première étape, nous avons analysé le texte en question, relevant des spécificités qui méritent d'être prises en compte et qui peuvent être mises en relation avec ce que produisent les enfants. Ensuite de quoi, nous nous sommes intéressées à trois facettes du travail de reconstruction effectué par les enfants :

- les difficultés que les élèves rencontrent en reformulant le texte, les erreurs ou confusions qu'ils peuvent commettre ;

- la façon dont ils collaborent dans ce travail de "coreconstruction », comment leurs tours de paroles se complètent ou se précisent ;

- la façon dont ils comprennent le texte dans sa complexité, mais aussi la façon dont ils le retiennent, dans sa forme même, avec les épisodes qui le constituent, et avec les formules qui le structurent - des formules dont nous verrons qu'elles sont tout à la fois relativement obscures, et pourtant essentielles dans ce qui constitue le plaisir de ce texte.

11 C'est en s'appuyant sur ces trois aspects de leur travail que nous revenons, dans une troisième partie, sur la question de la pratique du langage en général.

\section{Un texte singulier}

\section{Un texte répétitif}

Une des particularités de ce texte est d'être l'histoire d'une répétition: Pierre répète l'histoire qui fut celle de son père. Cette répétition en outre n'est pas la première, si l'on en croit ce qu'indique la mère à la fin, qui ne fait peut-être pas référence à d'autres intrusions dans le jardin de Monsieur Grégory, mais en tous les cas à d'autres mises en 
danger : «Pierre a encore perdu ses vêtements et ses chaussures. C'est la deuxième fois, cette semaine!»

Ou plutôt Pierre répète le début de l'histoire, il répète la transgression qui a conduit à la mort de son père, mais lui en sort vainqueur - apparemment du moins. L'une des difficultés de ce texte est de savoir comment interpréter cette issue où il «s'effondre, ferme les yeux et s'endort, épuisé ». Est-ce le repos du vainqueur? A-t-il vaincu le danger? Ou bien son épuisement doit-il être pris comme une sorte de petite mort, rejouant celle de son père pour mieux l'expier ou mieux l'exorciser. Répéter la scène du meurtre du père recèle nécessairement des enjeux importants pour le petit lapin, mais aussi pour tout un chacun qui entend l'histoire: comme c'est le cas sans doute de nombreux contes, cette histoire trouve là un sens, que les enfants reconstituent plus ou moins consciemment, mais qui joue un rôle crucial dans leur réception. Cependant, parce que le sens de cette répétition est obscur (expier, exorciser, dépasser, venger, etc.), et aussi parce que les différentes scènes qui se reproduisent peuvent se mélanger, l'ensemble du dispositif narratif s'en trouve fortement complexifié.

\section{Un texte complexe}

14 À ce premier niveau de complexité s'en ajoutent d'autres. L'ordre d'enchainement des évènements relève en effet d'une logique peu transparente, avec de nombreux sousscripts dont on voit mal à priori la cohérence :

- Après le festin du petit lapin dans le jardin, alors qu'il en était à manger du persil pour «digérer calmement», on le retrouve en train de «terminer tranquillement un concombre ".

- Après qu'il a entendu le "bruit étrange et inquiétant », le texte fait une sorte de retour en arrière pour décrire ce qui a conduit Monsieur Grégory à produire ce bruit effrayant : il "vient de l'apercevoir ", il «se lève d'un bond, et court vers Pierre en hurlant », ce qui au demeurant ressemble moins à un « bruit » qu'à des paroles.

- Les trois épisodes qui suivent, celui de la perte des chaussures, celui des groseilles, celui de l'arrosoir, marquent sans doute une progression dans la crise (il perd ses chaussures, il perd son gilet, il est mouillé et gelé), mais sont presque trop pittoresques pour s'inscrire dans une dynamique, avec à chaque fois un détail qui arrête la narration : les choux et les pommes de terre, le plant de groseilles et le bouton, l'arrosoir et l'eau glacée.

- L'épisode de la chatte blanche est particulièrement opaque et parait en rupture avec le récit: on ne sait quel est son rôle, sinon qu'il y est question de désir (voir l'histoire du poisson ${ }^{2}$ ) et qu'il forme à lui seul une scène de mise à mort reproduisant en miniature celle $\mathrm{du}$ père, et celle qui menaçait Pierre. En outre, cette scène est à la fois prise dans la dynamique de la course frénétique ( La course folle reprend de plus belle; il cherche désespérément à sortir du potager. ») et traitée comme un moment de suspension : la chatte «observe » le poisson rouge, et « Pierre n'ose pas la déranger ».

- Alors qu'il a été indiqué deux fois que Pierre ne trouvait pas la sortie, on le retrouve hors du jardin sans qu'ait été expliqué comment il est sorti.

- Il s'endort deux fois à la fin : en arrivant, épuisé, puis quand il est mis au lit par sa mère qui lui donne une tisane pour qu'il " passe une bonne nuit ». L'effet est que la mère réagit elle aussi en deux temps à l'aventure de son fils, la première fois «se demandant » et la seconde «ne sa(chant) pas ». 
La conclusion même de l'histoire est obscure, ne serait-ce que parce que personne ne sait vraiment ce que la mère a pu effectivement comprendre de ce qui s'est passé. Nous supposons qu'elle a partiellement compris puisqu'elle indique qu'il s'agit de la deuxième incartade de Pierre ; en outre, nous pouvons difficilement imaginer qu'elle n'ait pas fait le lien avec sa mise en garde du matin. Pourtant le texte se clôt sur la double interrogation de la mère, et par voie de conséquence sur celle du lecteur-auditeur.

L'identification des actants est en outre parfois ambigüe, en tous les cas entre Monsieur Grégory et Pierre. Dans les deux extraits ci-dessous, nous avons à chaque fois deux «il » qui se suivent et qui n'ont pas le même référent : «C'est monsieur Grégory qui vient de l'apercevoir alors qu'il est en train de planter des jeunes choux. Il se lève d'un bond [...] »; "Cependant, il ne bouge pas car monsieur Grégory approche. Il le cherche attentivement, partout ». Dans le même ordre d'idée, la formule « Petit voleur de lapin! » employée par Monsieur Grégory peut s'interpréter comme signifiant que le lapin est un petit voleur, mais pourrait aussi désigner celui qui vole les lapins - c'est-à-dire plutôt Monsieur Grégory lui-même qui n'en est pas à son premier lapin. Cette confusion est au demeurant redoublée par une autre, relative à l'interprétation du déterminant «son»: «Vous pouvez jouer dans le pré mais, surtout, surtout n'allez pas dans le jardin de monsieur Grégory ! Votre pauvre père n'en est jamais revenu! Madame Grégory a fait de lui un civet pour son repas ». Ce déterminant peut référer aussi bien à Monsieur Grégory (dans une interprétation sexiste faisant de Madame Grégory la cuisinière naturelle de son époux - interprétation qui est le plus souvent retenue par les enfants) ou à une Madame Grégory gourmande qui aurait profité de l'aventure pour se concocter un joli repas.

Que le texte soit opaque n'est pas nécessairement un défaut, au demeurant la plupart des points d'opacité qui viennent d'être mis en évidence sont aussi les points qui confèrent au texte une forme littéraire et une esthétique auxquelles les enfants sont clairement sensibles. Sur ce point, les positions s'opposent, avec d'un côté ceux qui considèrent que les textes à proposer aux enfants, quand ils doivent être ensuite racontés, doivent être d'une opacité réduite (Canut \& Vertalier, 2012), de l'autre côté des défenseurs de l'obscurité, qui en font une mesure du caractère littéraire des textes (Tauveron, 1999).

Quoi qu'il en soit, lorsque certains enfants restituent le récit de façon obscure ou discontinue, ou lorsqu'ils éprouvent des difficultés à le restituer, c'est en partie parce que le texte ne les aide pas à le faire, et en partie parce que ce faisant ils prennent effectivement la mesure de ce qui se joue dans ce texte. Et quand d'autres enfants arrivent à reconstituer des liens logiques et à différencier les protagonistes, ce n'est pas directement à cause de ce que dit le texte explicitement : cela suppose de leur part un travail implicite d'inférences complexes. Ce n'est pas le moindre des intérêts de ce corpus que de nous donner à observer le cheminement interprétatif mis en œuvre. Par là nous espérons prendre la mesure - au moins partiellement - des compétences en compréhension qui sont les leurs, et par-delà leurs compétences langagières qu'ils mobilisent pour dire ce qu'ils comprennent.

\section{Une reformulation parmi les reformulations}

19 Il est attendu que les enfants restituent l'histoire et donc d'une certaine façon la répète. De ce point de vue un autre aspect du texte mérite d'être pris en considération, qui est le fait qu'il s'inscrive lui-même dans une série de textes dont il est la restitution. Raconter non pas une histoire mais un texte racontant une histoire, ou plutôt la reracontant après 
d'autres versions ${ }^{3}$. Le dispositif pédagogique mis en œuvre favorise d'une certaine façon cette démultiplication : les enfants, qui racontent, intègrent dans leur restitution le texte qui leur a été lu, sans doute plusieurs fois, et qui leur a aussi peut-être été - dans certaines classes - raconté par l'enseignant; et pour ce faire il est prévu qu'ils puissent s'appuyer librement sur les restitutions des autres enfants, quitte à les répéter ou en tous les cas à les reformuler à loisir. Une telle structure en abyme des répétitions n'est pas en soi négative, toute parole procédant à des degrés divers de répétitions de cet ordre. Il parait indispensable en revanche de la prendre en compte pour entendre ce qui se joue dans les restitutions des enfants.

\section{Les enfants au travail}

\section{Difficultés}

Lorsque l'on observe la façon dont les enfants reformulent l'histoire, nous voyons bien qu'ils ont affaire aux opacités qui viennent d'être mises en évidence. Cela se manifeste notamment pour ce qui concerne l'identification des actants d'une part, leur rôle dans les actions d'autre part.

Ainsi trouve-t-on d'abord un certain nombre de cas où les personnages se confondent, notamment des cas où le même pronom réfère successivement à Pierre et à Monsieur Grégory, comme dans l'extrait suivant.

Tableau 1. Pronom 1 : Pierre ou Grégory

\begin{tabular}{|l|l|l|l|}
\hline Séance & $\mathbf{N}^{\mathbf{0}}$ & Loc & Interventions \\
\hline 3 & 115 & Malo & monsieur Grégory \\
\hline 3 & 116 & Marianne & et après il mange des petits légumes \\
\hline 3 & 117 & Malo & il est en train de planter ses choux \\
\hline
\end{tabular}

De même dans l'extrait ci-dessous de Mourad (séance 1), où l'on peut hésiter pour décider si c'est M. Grégory ou Pierre qui s'est « fait entendu ».

Tableau 2. Pronom 2 : Grégory ou Pierre

\begin{tabular}{|l|l|l|l|}
\hline Séance & $\mathbf{N}^{\mathbf{0}}$ & Loc & Intervention \\
\hline 1 & 45 & Mourad & $\begin{array}{l}\text { et après Pierre quand il était glacé/ il a fait a/ il a fait a/ atchoum/ il a } \\
\text { éternué et monsieur Grégory il s'est fait/ il s'est fait entendu/ monsieur } \\
\text { elle dit mais qu'est-ce qu'il fait mon fils/ pourquoi il y a pas ses habits/ } \\
\text { dis donc et il va avoir froid et après elle donne une tisane/ une tisane } \\
\text { avec quelque chose à manger et la mère et ses trois petits/ Pierre/ } \\
\text { Romarin il a peur/ et Pierre il dit rien à sa maman }\end{array}$ \\
\hline
\end{tabular}


Nous relevons aussi d'autres confusions intéressantes.

Tableau 3. Pronom 3 : maman ou Madame Grégory

\begin{tabular}{|l|l|l|l|}
\hline Séance & $\mathbf{N}^{\mathbf{0}}$ & Loc & Intervention \\
\hline 10 & 14 & Ted & $\begin{array}{l}\text { eh ben la maman elle avait dit de pas aller dans le jardin de monsieur } \\
\text { Grégory/ sinon elle va lui en faire un pâté }\end{array}$ \\
\hline
\end{tabular}

On peut supposer ici que le second pronom « elle » renvoie plutôt à Madame Grégory, bien que son nom n'apparaisse pas. On le suppose parce que c'est elle et non la mère qui a fait un pâté. La confusion est double dans la mesure où Madame Grégory n'a pas fait de pâté (du moins dans cette version), mais un civet : c'est Monsieur Grégory qui parle de pâté plus tard lorsqu'il menace Pierre et dit cette phrase " Je vais te transformer en pâté " - une menace qui est de fait la répétition de la menace proférée par la mère. Ce « elle » télescope ainsi deux moments du texte - deux menaces, et aussi deux scènes de mise à mort - et trois personnages : mais ces deux moments sont de fait des répétitions l'un de l'autre, et on a vu ci-dessus que le texte télescopait quant à lui le père, Monsieur Grégory et Madame Grégory autour de cette affaire de repas («son repas »). Ainsi, la question se pose de savoir si Ted a employé le mauvais pronom personnel pour désigner Monsieur Grégory ou s'il souhaitait faire référence à Madame Grégory, utilisant alors un pronom qui y renvoie sans en préciser le référent. Cette ambigüité est fréquente dans les activités de narration à l'oral chez l'enfant (Vion \& Colas, 1998; Weck, 1991) et conduit comme ici à de multiples interprétations possibles. Il n'empêche qu'elle est liée dans ce cas à ce qui constitue l'un des nœuds de l'histoire restituée.

Il y a par ailleurs massivement des confusions entre Pierre et son père, comme dans l'échange suivant.

Tableau 4. La course : Pierre ou son papa

\begin{tabular}{|l|l|l|l|}
\hline Séance & $\mathbf{N}^{\mathbf{0}}$ & Loc & Interventions \\
\hline 3 & 47 & Melwan & il a couru tout vite \\
\hline 3 & 48 & M & qui \\
\hline 3 & 49 & Viviane & Pierre \\
\hline 3 & 50 & M & on ne parle pas de Pierre pour le moment \\
\hline 3 & 51 & Melwan & on parle de son papa \\
\hline 3 & 52 & Reda & il a couru \\
\hline 3 & 53 & Sam & vite \\
\hline 3 & 54 & Reda & il a couru tout vite et après monsieur Grégory l'a attrapé/ il l'a ramené \\
\hline
\end{tabular}


Ou dans la séance 12 , sous forme non pas de confusion mais de réparation : «ce que je voulais dire/ eh ben Pierre/ je veux dire son le papa de Pierre/ eh ben il est allé dans le potager/ il est allé manger et monsieur Grégory il a fait un civet de lapin au papa de Pierre " (Jean, S12-62). On a au demeurant dans cette intervention de Jean une nouvelle confusion : il ne s'agit pas de faire un civet pour le papa de Pierre, mais de faire du papa de Pierre un civet. Ici, Monsieur Grégory et le papa se confondent. Surtout la confusion s'inscrit dans la perspective d'une autre question dont on va voir juste après l'importance, qui est une affaire de diathèse, relative à qui fait quoi, et en l'occurrence à qui mange, qui est mangé, et qui fait manger.

Tous ces apparents ratés prennent plus de sens si l'on considère les relations compliquées qui lient les personnages dans l'histoire, avec d'une part le fait que l'aventure de Pierre répète celle de son père, d'autre part le fait qu'en bravant l'interdit posé par sa mère d'entrer dans le jardin, Pierre brave par la même occasion l'interdit posé par les Grégory qui ne veulent pas de lapins dévorant leurs légumes: de ce point de vue, Monsieur et Madame Grégory incarnent des figures parentales, coïncidant dès lors avec les parents de Pierre, par-delà le brouillage dû au fait que le père lui-même a bravé l'interdit.

Concernant maintenant le rôle des actants dans les différentes actions entreprises, on trouve aussi des confusions intéressantes.

Tableau 5. Diathèse 1 : sortir par les oreilles

\begin{tabular}{|l|l|l|l|}
\hline Séance & $\mathbf{N}^{\mathbf{0}}$ & Loc & Interventions \\
\hline 2 & 87 & M & il le sort de l'arrosoir monsieur Grégory? \\
\hline 2 & 88 & Sandra & non/ il le voit \\
\hline 2 & 89 & M & mais Kaïss il a dit/ \\
\hline 2 & 90 & Gaël & il le sortait par les oreilles// \\
\hline 2 & 91 & M & vous êtes d'accord// \\
\hline 2 & 92 & Gaël & \\
\hline 2 & 93 & M & vas-y Gaël \\
\hline 2 & 94 & Gaël & il sort de l'arrosoir// \\
\hline
\end{tabular}

Dans cet échange, on passe de Monsieur Grégory faisant sortir Pierre de l'arrosoir ( il le sortait ») à Pierre sortant de par lui-même (« il sort »). Or dans les faits, les deux options sont correctes : même si Monsieur Grégory ne sort pas Pierre par les oreilles, c'est bien sa présence qui fait sortir Pierre. 
30 Il $\mathrm{y}$ a beaucoup d'autres cas où les enfants vont ainsi jouer sur la diathèse des verbes, brouillant les cartes entre qui est l'agent, qui est la cause, qui est le patient, qui est le bénéficiaire. C'est même peut-être la principale source de maladresses syntaxiques, quand les actants se mélangent, comme dans l'extrait suivant, où le père patient devient agent («Il a fait un civet»), selon un emploi de «faire» qui pourrait ressembler à « servir » : le lapin a servi de civet pour monsieur Grégory, cela lui a fait un beau civet.

Tableau 6. Diathèse 2 : faire un civet

\begin{tabular}{|l|l|l|l|}
\hline Séance & $\mathbf{N}^{\mathbf{0}}$ & Loc & Intervention \\
\hline 10 & 109 & Ewan & $\begin{array}{l}\text { et la maman elle leur a dit va pas/ allez pas dans le jardin de monsieur } \\
\text { Grégory }\end{array}$ \\
\hline
\end{tabular}

31 De même dans l'extrait suivant, avec la préposition " pour ", où l'on hésite à interpréter « le papa » comme le thème (pour ce qui concerne le papa) ou comme le bénéficiaire :

Tableau 7. Diathèse 3 : faire un civet pour

\begin{tabular}{|l|l|l|l|}
\hline Séance & $\mathbf{N}^{\mathbf{0}}$ & Loc & Intervention \\
\hline 2 & 109 & Sima & la femme de monsieur Grégory fait une civet pour le papa// \\
\hline
\end{tabular}

On peut rapprocher ces hésitations d'un autre effet de diathèse concernant cette fois la « course folle», qui selon les cas « reprend» ou est reprise, sans que cela corresponde cette fois à une maladresse syntaxique, mais bien à un jeu d'ergativité largement répandu dans les langues :

Tableau 8. Diathèse 4 : reprendre

\begin{tabular}{|l|l|l|l|}
\hline Séance & $\mathbf{N}^{\mathbf{0}}$ & Loc & Interventions \\
\hline 3 & 246 & Yannick & il reprend la course folle \\
\hline 3 & 247 & Malo & la course folle reprend \\
\hline
\end{tabular}

33 On voit que si les relations actancielles sont brouillées, cela tient sans doute parfois à une maladresse syntaxique des enfants, mais aussi parfois à une réelle adresse de ces enfants, et en tous les cas à un fonctionnement linguistique général, correspondant notamment au fait que les verbes eux-mêmes admettent plusieurs schémas actanciels (cf. faire, sortir et reprendre ci-dessus). Or cette confusion nous renseigne elle aussi sur ce qui se produit dans l'histoire de Pierre, dans laquelle il n'y a pas vraiment d'agent, de bénéficiaire ou de patient, mais des actants qui se défendent et se protègent les uns des autres : si le papa a été tué et si Pierre est menacé ce n'est pas tellement parce que Monsieur Grégory est gourmand, c'est d'abord parce que Pierre et le papa sont gourmands, et qu'il faut bien que Monsieur Grégory se protège de cette gourmandise pour lui menaçante. Qui est 
l'agresseur? Qui est la victime ? À qui profite le crime ? Ce sont ces questions qui sont derrière la question de l'agent, du patient et du bénéficiaire. Et elles sont au cœur de l'histoire.

\section{La coconstruction : reconstituer/reformuler/parler}

Lors de l'activité de restitution, les enfants ne se contentent pas de juxtaposer des informations sans relation, comme on aurait pu le craindre. Même si cela arrive, notamment lorsque l'enseignant les interpelle chacun à son tour, décidant dès lors de l'ordre d'intervention, la plupart du temps les enfants s'appuient sur les propos les uns des autres, coopérant ainsi pour coconstruire cette restitution.

Pour s'appuyer sur le dire de l'autre

Tableau 9. Répétitions et rebonds

\begin{tabular}{|l|l|l|l|}
\hline Séance & $\mathbf{N}^{\mathbf{o}}$ & Loc & Interventions \\
\hline 2 & 49 & $\mathrm{M}$ & monsieur Grégory il a dit par mes/ \\
\hline 2 & 50 & Sandra & mes moustaches arrête petit voleur/ je vais te transformer en pâté \\
\hline 2 & 51 & El(s) & te transformer en pâté \\
\hline 2 & 52 & Sandra & comme ton père \\
\hline
\end{tabular}

C'est ici l'ensemble des élèves qui répètent (avec jubilation) la formule répétée par Sandra, ce qui sert alors à Sandra de tremplin pour enchainer.

\section{Pour continuer}

Dans l'extrait ci-dessous, après avoir annoncé l'histoire par le présentatif « il était une fois » et après avoir complété la liste des personnages, les élèves continuent en s'appuyant les uns sur les autres pour former une seule longue phrase qui va décrire l'action, leur donnant l'occasion d'utiliser une relative, une infinitive, mais aussi du discours indirect (plus ou moins stabilisé, avec un conditionnel) et une causale (soulignés dans l'extrait).

Tableau 10. Phrase à 4 voix

\begin{tabular}{|l|l|l|l|}
\hline Séance & $\mathbf{N}^{\mathbf{0}}$ & Loc & Interventions \\
\hline 3 & 13 & Viviane & des petits lapereaux \\
\hline 3 & 14 & Malo & qui vivaient dans un vieux terrier/vieux pin centenaire \\
\hline 3 & 15 & Melwan & qui vivaient avec sa maman \\
\hline 3 & 16 & Marianne & oui/ sa maman il a dit/ vous pouvez \\
\hline
\end{tabular}




\begin{tabular}{|l|l|l|l|}
\hline 3 & 17 & Malo & d'aller dans le pré/ d'aller dans le pré \\
\hline 3 & 18 & Melwan & et puis son papa il reviendrait jamais parce qu'il s'est fait manger \\
\hline 3 & 19 & Malo & dans le jardin \\
\hline
\end{tabular}

\section{Pour compléter et expliquer}

Tableau 11. Variations sur atchoum

\begin{tabular}{|l|l|l|l|}
\hline Séance & $\mathbf{N}^{\mathbf{0}}$ & Loc & Interventions \\
\hline 2 & 287 & Soliman & il est malade à cause de l'eau froide \\
\hline 2 & 288 & Elise & a:::tchoum \\
\hline 2 & 289 & Sandra & il éternue \\
\hline 2 & 290 & Soliman & il éternuait \\
\hline 2 & 291 & Alice & $\begin{array}{l}\text { il est malade parce qu'il est allé dans le jardin de monsieur Grégory et } \\
\text { qu'il a tout mangé et il s'est caché dans l'arrosoir }\end{array}$ \\
\hline
\end{tabular}

37 Au plan du matériel langagier, ces précisions sont l'occasion (en tous les cas pour Alice) d'utiliser une causale et deux coordinations.

Pour affiner... jusqu'à inverser

Tableau 12. Inversion $1:$ de ne pas arriver à arriver

\begin{tabular}{|l|l|l|l|}
\hline Séance & $\mathbf{N}^{\mathbf{0}}$ & Loc & Interventions \\
\hline 2 & 69 & Sima & il XXX \\
\hline 2 & 70 & Sandra & il coince son gilet \\
\hline 2 & 71 & M & attends elle va le dire/ vas-y Sima elle t'a un peu aidée \\
\hline 2 & 72 & Sima & \\
\hline 2 & 73 & M & redis-lui Sandra \\
\hline 2 & 74 & Sandra & il essaie d'enlever son gilet \\
\hline 2 & 75 & M & vas-y Sima \\
\hline 2 & 76 & Sima & et il arrivait pas à enlever son gilet \\
\hline
\end{tabular}


Dans cet extrait, le verbe «enlever » permet de passer du blocage de départ (70 : gilet coincé) à sa résolution (77: gilet enlevé). Proposé par Sandra (74), il est d'abord repris sous forme négative par Sima (76) comme une reformulation de « coincer son gilet » (il coince son gilet $=$ il n'arrive pas à l'enlever). Et il est ensuite réinvesti par Alice (77) pour dire ce qui permet au lapin de se décoincer : il se déshabille, et laisse son gilet dans le filet. Dans l'opération, "enlever» a changé de sens et d'orientation, passant de « décoincer » (enlever du filet) à « se déshabiller » (enlever de soi). Un autre exemple de précision par inversion, toujours dans la séance 2, est d'autant plus joli qu'il marque la victoire de Pierre :

Tableau 13. Inversion 2 : de faillir à ne pas

\begin{tabular}{|l|l|l|l|}
\hline Séance & $\mathbf{N}^{\mathbf{0}}$ & Loc & Interventions \\
\hline 2 & 313 & M & et donc// \\
\hline 2 & 314 & Sandra & il a failli se faire avoir \\
\hline 2 & 315 & Gaël & mais il s'est pas fait avoir \\
\hline
\end{tabular}

\section{Pour construire le récit et l'ordonner}

On voit bien comment dans les extraits de 13 à 19 du Tableau 13 ci-dessous, les élèves coopèrent pour construire le récit dans son enchainement, avec des interventions scandées par « et après » qui accumulent dans l'ordre les évènements du texte (de 1 à 6 évènements selon les interventions).

Tableau 14. Reconstruction ordonnée

\begin{tabular}{|l|l|l|l|l|}
\hline Séance & $\mathbf{N}^{\mathbf{0}}$ & Loc & Interventions & $\begin{array}{l}\text { Nombre } \\
\text { d'évènements } \\
\text { mentionnés }\end{array}$ \\
\hline 6 & 12 & Marco & $\begin{array}{l}\text { oui// et après il va dans le jardin et après euh tout il } \\
\text { Grégory qui approche et après euh et après Pierre } \\
\text { qui dit il court }\end{array}$ & 4 \\
\hline 6 & 13 & Emilie & $\begin{array}{l}\text { et euh monsieur Grégory il dit sale voleur/ je vais } \\
\text { t'attraper et te transformer en pâté }\end{array}$ & 1 \\
\hline 6 & 14 & Leslie & $\begin{array}{l}\text { et après il perd une chaussure dans les dans les } \\
\text { choux et une chaussure dans les pommes de terre }\end{array}$ & 1 \\
\hline
\end{tabular}




\begin{tabular}{|l|l|l|l|l|}
\hline 6 & 15 & Daly & $\begin{array}{l}\text { il s'a accroché son bouton de gilet dans les plants de } \\
\text { groseilles }\end{array}$ & 1 \\
\hline 6 & 16 & Frédéric & $\begin{array}{l}\text { et après les oiseaux ils disent vite vite sauve-toi il y a } \\
\text { monsieur Grégory qui vient }\end{array}$ & 1 \\
\hline 6 & 17 & Emilie & $\begin{array}{l}\text { après il a trouvé une solution/ il enlève sa veste et il } \\
\text { va dans la remise pour aller dans l'arrosoir et il a fait } \\
\text { une erreur parce que l'eau elle était glacée// }\end{array}$ & 3 \\
\hline 6 & 18 & Leslie & $\begin{array}{l}\text { et au bout d'un moment il éternue parce que l'eau } \\
\text { elle était trop froide// après monsieur Grégory il } \\
\text { l'entend et il reprend sa course folle }\end{array}$ & 3 \\
\hline 6 & 20 & $\begin{array}{l}\text { Marco } \\
\text { et après il passe en dessous de ses jambes de } \\
\text { monsieur Grégory } \\
\text { monsieur Grégory il se retourne/ et après il } \\
\text { s'approche de l'arrosoir/ et après il reprend cette } \\
\text { course folle/ et après il a trouvé la porte }\end{array}$ & 1 \\
\hline et après il va dans l'arrosoir et l'eau il était tellement \\
\hline
\end{tabular}

\section{Pour combler les trous logiques}

On note dans la réplique 20 de l'exemple précédent que Marco est revenu en arrière au lieu de continuer le récit : l'enfant reprend ce qui a été dit dans l'intervention 17 (Pierre va dans l'arrosoir et l'eau est glacée) et enchaine sur l'énoncé 18 (il éternue ; Monsieur Grégory l'entend), qu'il va alors expliciter, en précisant ce qui n'était pas dit dans 18 et qui explique la course folle, à savoir le fait que Monsieur Grégory s'est approché de l'arrosoir. Ainsi est-il remonté à l'énoncé 17 pour relancer une boucle. C'est donc bien le sens qui ici est en jeu, et en l'occurrence les raisons de cette nouvelle fuite. Cette boucle est au demeurant l'occasion pour les élèves de déployer les subordinations avec des relatives (en 12 et 16 associée à une forme présentative), une finale (« pour » en 17), deux causales (« parce que » en 17 et 18) et une consécutive (« tellement que » en 20).

\section{Pour préciser les circonstances}

On se réfère ici aux travaux de D. Davidson (1967) montrant que le propre des évènements est qu'ils puissent être décrits par n'importe laquelle des circonstances qui les a déterminés: D. Davidson le démontre à partir d'exemples de propositions décrivant l'assassinat de César (Brutus plante le couteau / Brutus tue César / Brutus trahit César / le sang gicle / Brutus lève la main, etc.) : toutes décrivent l'assassinat, la différence étant seulement une différence de "grain" dans la description. Or cette variation est cruciale pour l'interprétation dans la mesure où elle permet de mettre en évidence telle circonstance, tel détail, telle motivation, telle finalité. Les reformulations des enfants jouent beaucoup sur cette variation de grain dans le rendu des évènements de l'histoire. 
Tableau 15. Circonstances 1 : détails, motivations, finalités

\begin{tabular}{|l|l|l|l|}
\hline Séance & $\mathbf{N}^{\mathbf{0}}$ & Loc & Interventions \\
\hline 3 & 155 & Marianne & il est prêt à l'attraper \\
\hline 3 & 156 & Malo & il veut le manger \\
\hline 3 & 157 & Sam & il veut le cuire \\
\hline 3 & 158 & Melwan & quand ils vont le transformer en pâté/ ils vont le cuire et puis \\
\hline 3 & 159 & Yannick & ils vont le manger \\
\hline 3 & 160 & Melwan & ils vont le cuire/ ils vont le couper et puis après ils vont le manger \\
\hline
\end{tabular}

43 Être prêt à attraper / vouloir manger / vouloir cuire / «aller » transformer en pâté / «aller » cuire / «aller » manger / «aller » couper - toutes ces formulations décrivent bien la situation particulière en jeu: une situation qui précède l'action, qui est donc caractérisée par ce qui est imminent («aller»), par une volonté («vouloir»), par une capacité (« être prêt»); et une situation dont la finalité est un enchainement d'actes (attraper, manger, cuire, transformer en pâté, cuire, manger, cuire, couper, manger) dont chacun dit le sens des autres. Ainsi, chacune de ces reformulations permet de comprendre la situation et de comprendre l'acte visé, dans ses modalités, comme dans ses motivations et ses finalités.

Nous en avons relevé plusieurs exemples dans la séance 3, qui sont sans doute induits par l'intervention de l'enseignant, qui a pour particularité d'utiliser comme relance la question du "pourquoi ». Nous relevons cependant des variations du même ordre, par exemple dans les échanges de la séance 1 , à propos d'un autre évènement du texte : la transgression de l'interdit de la mère, qui peut être décrite avec des verbes comme désobéir, ou avec des verbes de parole restituant l'énoncé de l'interdit, ou directement avec des négations, mais aussi avec tout verbe décrivant cette transgression à l'œuvre ( passer, aller, venir, rentrer).

Tableau 16. Circonstances 2 : aller, venir, passer, jouer

\begin{tabular}{|l|l|l|l|}
\hline Séance & $\mathbf{N}^{\mathbf{0}}$ & Loc & Interventions \\
\hline 1 & 5 & Oudriss & $\begin{array}{l}\text { après Pierre/ après il a dit/ après il a fait atchoum/ et après il a fait et } \\
\text { après Pierre et après Pierre il a été dans le potager et la maman } \\
\text { et la maman elle a dit ne passez pas dans le potager }\end{array}$ \\
\hline 1 & 6 & Sohaib & $\begin{array}{l}\text { mais comme Pierre il était le plus intré/ le plus trépide de la famille/ il } \\
\text { voulait venir dans le potager et il est quand même venu/ il est rentré sans } \\
\text { problèmes/ et après il a dit/ il a dit c'était un potager de rêve }\end{array}$ \\
\hline
\end{tabular}




\begin{tabular}{|l|l|l|l|}
\hline 1 & 7 & Sihène & $\begin{array}{l}\text { eh bien Pierre/ il est/ il est allé dans le jardin de monsieur Grégory et } \\
\text { après monsieur Grégory il a couru/ couru et Pierre il était tellement } \\
\text { fatigué eh bien/ et monsieur Grégory il a couru/ couru et Pierre/ Pierre il } \\
\text { était fatigué }\end{array}$ \\
\hline 1 & 8 & Daoud & $\begin{array}{l}\text { eh bien la mère elle a dit vous ne pouvez jouer que dans le pré et elle a dit } \\
\text { n'allez surtout pas dans le potager }\end{array}$ \\
\hline
\end{tabular}

Il s'agit ici de comprendre ce qui est l'enjeu de l'interdit, mais aussi ce que représente ce jardin interdit, à la fois extérieur aux lieux des lapins (« dans le pré »), limite (" passer »), lieu clos ( «aller dans », « rentrer »), et lieu origine où tout se joue (« venir », « rentrer »). Jouer sur les grains de la description est bien un type de reformulation, mais qui ne relève pas de la paraphrase, puisqu'elle procède plutôt par précision et explicitation, et qui ne relève pas non plus de la correction ou de la rectification, mais bien de la compréhension : il s'agit de faire émerger les enjeux.

$\mathrm{Au}$ travers de l'ensemble de ces collaborations entre les enfants, on observe ainsi les enfants apprendre à s'appuyer sur les dires des autres, mais aussi travailler la compréhension du texte, y compris dans ses dimensions les plus obscures.

\section{Raconter pour comprendre/raconter pour entendre}

47 On a vu comment les reformulations permettent aux enfants de travailler à comprendre collectivement le texte. Comprendre le texte, c'est comprendre la répétition qui se trouve au cœur de l'histoire, entre mort du père et transgression du fils, et on a vu comment les essais et les erreurs sur les pronoms y participent. C'est aussi comprendre que les intérêts des uns n'étant pas ceux des autres, il n'y a pas nécessairement de méchant et de gentil, de coupable et de victime. Cela renvoie aux jeux de diathèse que l'on a mis en évidence, dans lesquels les relations actancielles se brouillent. L'histoire est de ce point de vue une forme d'illustration de la chaine du vivant, avec tout le monde mangeant quelque chose Monsieur Grégory qui mange le lapin qui mange les légumes. Là aussi les échanges entre les enfants, leurs erreurs et leurs reformulations manifestent leur réflexion sur cette question. Par exemple, dans l'intervention de Yannick ci-dessous, on entend sans doute une erreur d'interprétation de la question dont Yannick n'a pas compris sur qui elle portait (problème d'anaphore encore), mais on entend surtout ce qui fonde cette erreur, à savoir le fait que Monsieur Grégory commet lui aussi un acte répréhensible, parallèle à celui que commet Pierre, l'un mangeant les lapins quand l'autre mange les choux.

Tableau 17. Retournement 1

\begin{tabular}{|l|l|l|l|}
\hline Séance & $\mathbf{N}^{\mathbf{0}}$ & Loc & Interventions \\
\hline 3 & 385 & M & quelle est la grosse bêtise qu'il a faite \\
\hline 3 & 386 & Viviane & il a désobéi \\
\hline 3 & 387 & Yannick & il a mangé les lapins \\
\hline
\end{tabular}


\begin{tabular}{|l|l|l|l|}
\hline 3 & 388 & Melwan & non/ il a mangé les choux de monsieur Grégory
\end{tabular}

De même dans l'échange qui suit, Malo veut sans doute tout à la fois contredire Clarissa et réaffirmer le présupposé de la question de l'enseignant (« ennemi» 䃈 «n'aime pas»), mais il n'ignore pas que Clarissa avait en tête un autre sens du verbe "aimer ", où il est question de goût et non pas d'amitié; et de ce point de vue, les deux réponses sont compatibles, le goût pour les lapins pouvant de fait aller de pair avec l'inimitié, puisqu'il faut que les lapins soient morts et passés à la casserole pour qu'on puisse les goûter.

Tableau 18. Retournement 2

\begin{tabular}{|l|l|l|l|}
\hline Séance & $\mathbf{N}^{\mathbf{0}}$ & Loc & Interventions \\
\hline 3 & 409 & M & pourquoi monsieur Grégory est l'ennemi des lapins \\
\hline 3 & 410 & Clarissa & parce qu'il aime bien les lapins \\
\hline 3 & 411 & Malo & il n'aime pas les lapins \\
\hline
\end{tabular}

On aboutit à une contradiction qui n'est pas loin du mot d'esprit : mot d'esprit sur le sens du mot aimer d'abord, mais aussi sur les ambivalences de l'amour, et sur les relations entre désir et destruction qui sont au cœur du récit. Avec de tels télescopages, on n'est pas, ou pas seulement, dans le ratage : on est dans un travail de compréhension du récit, de ses non-dits et des difficiles questions existentielles qui le constituent sans être formulées explicitement.

Cependant, quand nous racontons une histoire ce n'est pas seulement pour comprendre, c'est aussi pour entendre un texte, et pour l'apprécier. Les épisodes opaques font partie de ce qui rend un texte appréciable. Or nous voyons bien comment les enfants se raccrochent à ces épisodes, qui loin de les faire fuir, les fascinent, et sur lesquels ils reviennent volontiers en boucle : l'épisode de la chatte, l'affaire de la camomille, les groseilles, l'arrosoir, les choux et les pommes de terre, et même la liste des légumes dans le "jardin de rêve $~^{4}$. Les enfants répètent aussi les mots et les formules du texte, $\mathrm{y}$ compris les plus opaques. Ils reprennent, avec une forme de délectation :

- des paroles : Quelle erreur !»; « Petit voleur de lapin!»; «Par mes moustaches !»;

- des expressions figées : « il reprend sa course folle »; « couru ventre à terre »;

- des effets de style : «l'une dans les choux et l'autre dans les pommes de terre »;

- et même des mots obscurs comme "intrépide» ou des expressions difficiles comme « perturbé par les émotions de la journée » qui sont répétés y compris par des enfants qui ne les connaissent manifestement pas et qui ne les ont sans doute pas tout à fait compris (voir « le plus intré/ le plus trépide de la famille » de Sohaib (S1-6) dans le Tableau 14). 


\section{De comprendre et entendre à apprendre à parler}

52 Pas plus que ne saurait l'être tout texte un peu construit, ce texte complexe, à la forme tout à la fois singulière et plaisante, ne peut être réduit au rôle de support pour l'apprentissage du langage. Le comprendre, l'entendre et l'apprécier sont des enjeux en soi. D'autant qu'il recouvre, comme nous l'avons vu, des questions essentielles, qu'elles soient d'ordre psychanalytiques (meurtre du père, transgression) ou philosophiques (les déterminations qui régissent les actes de chacun), qui ne dépendent pas tant de connaissances culturelles que de l'expérience ordinaire de tout être humain, aussi petit soit-il. Le réduire à être un simple moyen pour une finalité autre qui serait l'apprentissage du langage n'aurait pas de sens.

53 Cependant maitriser le langage ne se réduit pas non plus à savoir utiliser des phrases et produire des discours. Parler c'est aussi comprendre et entendre: comprendre et entendre l'autre, comprendre et entendre les textes. De ce point de vue on reste bien dans l'apprentissage du langage.

54 Parler c'est aussi citer, répéter et reformuler ce que l'autre dit et ce qui se trouve dans tous les textes dans lesquels on s'inscrit lorsque l'on parle. Que les enfants répètent (ce que les autres disent ou ce que le texte dit) ne saurait être pris ni comme un défaut ni comme un manque d'autonomie linguistique: répéter, reformuler et par ce biais s'approprier sont des activités centrales dans la pratique du langage et il faut au contraire que les enfants réalisent le caractère dialogique de leur propre parole et qu'ils puissent ainsi jouer de ces répétitions et de ces reformulations pour construire leur parole authentique, tissée d'autres paroles. Le dispositif de restitution de ce texte favorise en tous les cas cette dimension de l'apprentissage du langage.

55 Il favorise aussi l'apprentissage d'aspects plus formels du langage: nous avons vu à différents moments comment il contribue à faire évoluer le langage des élèves : le travail de formulation effectué participant à l'acquisition ou à la mise en pratique d'une maitrise des constructions linguistiques. De fait, à chaque reformulation, les élèves reprennent des éléments du texte ou des éléments de leurs pairs, mais aussi ajoutent des éléments : on l'a noté au fur à mesure dans les exemples cités avec les différentes structures syntaxiques complexes que ces enchainements permettaient (relatives, discours indirect, circonstancielles, etc.), et avec le jeu sur les diathèses, sur le sens des mots utilisés, ou sur ce que l'on a appelé le "grain » des formulations. Y compris lorsqu'ils se contentent de reprendre, le résultat est productif ${ }^{5}$. C'est aussi une occasion pour l'enfant d'utiliser luimême la forme reprise, de se l'approprier et de l'adapter à ses propres propos.

Y compris aussi lorsqu'ils se perdent dans les pronoms et dans les diathèses, $\mathrm{y}$ compris lorsqu'ils travaillent sur les enchainements, listent sans lien, reconstituent des chaines partiellement cohérentes, retracent des boucles, ils trouvent là l'occasion d'expérimenter problèmes d'anaphores, schémas actanciels et effets d'enchainements lorsqu'ils sont complexes et qu'ils peuvent de fait obéir à plusieurs logiques : on a vu que les problèmes qui se posent aux enfants sont des problèmes inscrits dans le texte lui-même.

57 Ainsi, pour ceux qui parlent, mais aussi pour ceux qui écoutent et entendent ces différentes reformulations, ce travail de restitution est l'occasion de faire des expériences de langage. Et ces expériences sont riches: les formes linguistiques que l'on a pu 
observer, que ce soit dans le texte raconté ou dans les restitutions des enfants, sont des formes complexes.

\section{Quels apports pour la formation des enseignants?}

Dans ce dispositif, l'enseignant est très en retrait, il intervient très peu, sauf pour poser des questions prédéfinies qui incitent les élèves à raconter dans un premier temps et à décrire les personnages dans un second temps. Nous avons vu cependant que le fait d'interroger les enfants l'un après l'autre ou de poser la question du pourquoi avait des effets importants sur ce qu'ils produisent. L'enseignant est donc plus actif que l'on pourrait le penser. À partir de là, la question de l'impact de l'étayage de l'adulte dans ce type d'activité reste entière : faut-il, lorsqu'il y a problème, laisser l'enfant ou les autres enfants ou le temps résoudre le problème, ou faut-il proposer l'offre langagière propre à le résoudre? Nous ne voulons pas trancher ici la question de savoir s'il faudrait plus d'étayage et si ce type de dispositif doit être généralisé dans tous types de contextes, quel que soit l'enseignant, et avec tous les élèves de cette tranche d'âge ${ }^{6}$. Nous pensons que le dispositif a en tous les cas l'avantage de nous permettre de mieux mesurer, grâce aux données qui nous sont livrées, ce qui est en jeu dans la compréhension d'un texte par des élèves non encore lecteurs, dans la restitution d'un tel texte, et aussi dans la maitrise du langage par les enfants de cet âge.

Cela nous conduit à proposer d'exploiter ces résultats sinon directement via ce dispositif en particulier, du moins en les intégrant dans la réflexion sur la formation des enseignants, de manière à ce que ces derniers puissent mieux appréhender chacun de ces enjeux, relatifs à la compréhension d'un texte et aux difficultés que les élèves peuvent rencontrer, à la coconstruction, à ses pratiques et à leurs effets, à la maitrise du langage et aux différentes formes qu'elle peut prendre. L'analyse de ce dispositif et de la façon dont il a pu être mis en œuvre laisse à penser qu'il est important que les enseignants reçoivent une formation sur les spécificités liées à l'activité de restitution d'un texte : un texte peut se raconter de plusieurs façons; tous les textes s'appuient sur d'autres textes; on peut restituer des épisodes, restituer la structure générale, restituer des formules, entendre les enjeux, entendre les non-dits, etc. ; raconter, re-raconter permet d'affiner la restitution et la compréhension (Vygotski, 1997) et permet aussi, en parlant ainsi l'écrit, de mieux entrer dans l'écrit. aient une connaissance fine des spécificités des textes utilisés et qu'ils soient entrainés à en faire l'analyse, pour mettre en évidence structure, obscurité, enjeux, non-dits, esthétique: ainsi pourront-ils mieux favoriser les interactions avec les élèves, mieux mesurer les difficultés, mieux comprendre ce qui se joue dans les restitutions des enfants.

De même, il serait important de donner aux enseignants une formation sur ce que peut être la coconstruction d'une restitution: parler ensemble en s'appuyant non pas seulement sur le texte d'une part, sur ce que dit l'enseignant d'autre part, mais aussi sur ce que disent les autres élèves. 
textes en reconstituant la façon dont les évènements s'y enchainent et les structures qui les organisent. Mais tout cela passe aussi par ce que nous avons retrouvé dans ce corpus : parler, c'est aussi savoir s'appuyer sur la parole de l'autre, savoir l'apprécier, la répéter et la reformuler.

Enfin, il serait sans doute utile de pouvoir prolonger la formation linguistique des enseignants, en les alertant toujours plus sur les effets de double sens (enlever et enlever, aimer et aimer), sur les effets de diathèse et les schémas actanciels (faire un civet du point de vue du lapin et faire un civet du point de vue du cuisinier, sortir et faire sortir un lapin ou des oreilles, reprendre selon que la course reprend ou qu'on la reprend), et sur les jeux de grain (quand manger, cuire et attraper peuvent décrire le même évènement dans ses différentes circonstances, ou quand désobéir, aller, passer, jouer, manger décrivent la même transgression) : des effets, des schémas et des jeux avec lesquels un enfant travaille quand il faut qu'il parle et qu'il comprenne.

\section{BIBLIOGRAPHIE}

BAUTIER, É. (2001). « Pratique langagière et scolarisation ». Revue française de pédagogie 137, p. 117-161. En ligne : https://www.persee.fr/doc/rfp_0556-7807_2001_num_137_1_2853.

CANUT, E. \& VERTALIER, M. (2012). «Lire des albums : quelle compréhension et quelle appropriation par les élèves de maternelle? ». Le français aujourd'hui 179, p. 51-66. En ligne : https:// www.cairn.info/revue-le-francais-aujourd-hui-2012-4-page-51.htm.

CUlioli, A. (1999). «Conditions d'utilisation des données issues de plusieurs langues naturelles ». In : Culioli, A., Pour une linguistique de l'énonciation, tome 2. Gap/Paris : Ophrys, p. 67-82.

DAVIDSON, D. (1967). « The logical form of action sentences ». In : Rescher, N. (ed.), The Logic of Decision and Action. Pittsburgh : University of Pittsburgh Press, p. 81-95.

DIATKINE, R. (1984). « La formation du langage imaginaire ». Le français aujourd'hui 68, p. 25-29.

FUCHS, C. (1982). La paraphrase. Paris : Presses universitaires de France.

FUCHS, C. (1994). Paraphrase et énonciation. Gap/Paris : Ophrys.

GARCIA-DEBANC, C. (2006). « Une méthodologie pour déterminer les objets effectivement enseignés : l'étude des reformulations dans l'interaction didactique. Étude de cas d'une séance conduite par un enseignant débutant en fin d'école primaire ». In : Schneuwly, B. \& ThévenazChristen, T. (dirs), Analyses des objets enseignés. Le cas du français. Bruxelles : De Boeck, p. 111-141. GÜLICH, É. \& котSCHI, T. (1987). « Les actes de reformulation dans la consultation La dame de Caluire ». In : Bange, P. (éd.), L'analyse des interactions verbales. La dame de Caluire. Berne : P. Lang, p. 15-81.

JAUBERT, M., REBIÈRE, M. \& PUjo, J. (2010). « Communautés discursives disciplinaires scolaires et formats d'interactions », Colloque international «Spécificités et diversité des interactions didactiques: disciplines, finalités, contextes ", Lyon, 24-26 juin. 
LE BOT, M.-C., SCHUWER, M. \& RICHARD, É. (dirs) (2008). La reformulation. Marqueurs linguistiques, stratégies énonciatives. Rennes : Presses universitaires de Rennes.

LENTIN, L. (2009). Apprendre à penser, parler, lire, écrire. Paris : Éd. ESF.

MARTINOT, C. (2010). « Reformulation et acquisition de la complexité linguistique ». Travaux de linguistique 61, p. 63-96. En ligne : https://www.cairn.info/revue-travaux-de-linguistique-2010-2page-63.htm.

PÉGAZ-PAQUET, A. (2009). « Quand la reformulation s'invite à l'école... ». Cahiers de praxématique 52, p. 81-108. En ligne : https://journals.openedition.org/praxematique/1385?lang=en.

PÉROZ, P. (2014). « "parce que son papa, madame Grégory a fait un civet, un civet avec le papa”. Sur les procédures d'apprentissage du lexique : reprise et reformulation en langage oral à l'école maternelle ", Congrès mondial de linguistique française, Berlin, 19-23 juil. En ligne : https:// www.shs-conferences.org/articles/shsconf/pdf/2014/05/shsconf_cmlf14_01221.pdf.

PÉROZ, P. (2016). « Apprentissage du langage oral à l'école maternelle. Mise en œuvre d'un modèle polylogal d'apprentissage du langage oral en moyenne et grande section de maternelle en 2010-2013 ». Pratiques. Linguistique, littérature, didactique 169-170. En ligne : https:// journals.openedition.org/pratiques/3100.

RABATEL, A. (éd.) (2010). Les reformulations pluri-sémiotiques en contexte de formation. Besançon : Presses universitaires de Franche-Comté.

STEUCKARDT, A. (2009). « Usages polémiques de la reformulation ». Recherches linguistiques 29, p. 55-74.

TAUVERON, C. (1999). « Comprendre et interpréter le littéraire à l'école : du texte réticent au texte proliférant ». Repères 19, p. 9-38. En ligne : https://www.persee.fr/doc/ reper_1157-1330_1999_num_19_1_2289.

VION, M. \& ColAS, A. (1998). «L'introduction des référents dans le discours en français : contraintes cognitives et développement des compétences narratives ». L'année psychologique 98 , p. 37-59. En ligne : https://www.persee.fr/doc/psy_0003-5033_1998_num_98_1_28609.

VOlteAu, S. (2015). « Analyse des reformulations dans les interactions orales : l'exemple d'une séquence portant sur l'écosystème en CM2 ». Corela HS-18. En ligne : https:// journals.openedition.org/corela/4045.

VYGOTSKI, L. (1997) [1934]. Pensée et Langage. Trad. du russe par F. Sève. Paris : La Dispute. WECK, G. de (1991). La cohésion dans les textes d'enfants. Étude du développement des processus anaphoriques. Neuchâtel : Delachaux et Niestlé.

\section{NOTES}

1. Elle s'appuie aussi en droit sur une connaissance de divers marqueurs de reformulation (Gülich \& Kotschi, 1987), mais le dispositif étudié ne se prête pas vraiment à leur mise en œuvre.

2. Noter aussi le choix du féminin dans la version lue aux enfants : dans le texte initial (voir cidessous) il s'agit d'un « chat blanc ».

3. Danger dans le potager est une réécriture d'une première version française intitulée quant à elle Pierre le lapin (datant de 1986), qui elle-même est la traduction en français du texte original anglais The Tale of Peter Rabbit datant de 1902. 
4. Exemple séquence 6 énoncés 41 à 51 . On retrouve dans cette énumération tout ce qui relève pour le lapin, mais pour le lecteur aussi, de la gourmandise et du plaisir pur : il s'agit d'un jardin de rêve.

5. Voir les exemples de répétition avec retournements dans les Tableaux 15 et 16 ou dans un autre registre l'exemple de la séquence $6,12-20$, citée Tableau 13, où chaque enfant reprend la formule « et après ».

6. Pour évaluer véritablement l'efficacité du dispositif, il faudrait poursuivre le travail de P. Péroz $(2014 ; 2016)$ analysant, statistiques à l'appui, ses effets sur la syntaxe et sur l'apprentissage du lexique pour l'ensemble des enfants concernés.

\section{RÉSUMÉS}

Après avoir décrit la structure et les obscurités de l'histoire Danger dans le potager que des enfants de maternelle doivent restituer collectivement, l'article parcourt un certain nombre d'exemples de verbalisations produites par les enfants, qui nous donnent à voir différentes façons que les enfants ont de s'appuyer sur le texte, mais aussi sur les dires de leurs pairs, pour construire ensemble la restitution. L'objectif est d'une part d'examiner les manifestations qui attestent de leur compréhension de l'histoire et de ses enjeux, et de leur plaisir à l'entendre et à la dire euxmêmes, et d'autre part de revenir à partir de là sur ce que signifie apprendre à raconter et plus largement apprendre à parler. La dernière partie de l'article vise à dégager pour la formation des enseignants, un certain nombre de pistes et d'outils linguistiques permettant de mieux tirer parti de ce que mettent en jeu les reformulations observées.

This paper first describes the structure and the misleading features of a French version of Peter Rabbit, which pre-school children are invited to tell in shared storytelling. Examples of the children's productions are examined, to show how the children rely on the text, but also on peer production, for their joint narrative. The aim of this paper is, on the one hand, to analyse the children's expression of their understanding of the story and its issues, as well as of the pleasure they take in hearing and telling it by themselves. On the other hand, this analysis will help emphasize the impact of story telling on language acquisition. This paper also aims at pointing to possible guidelines and linguistic tools for teacher training in order to help teachers take advantage of their observation of the children's repetition and recasts of the story.

\section{INDEX}

Mots-clés : reformulation, narration, anaphore, diathèse, formation des enseignants

Keywords : reformulation, narrative, anaphora, diathesis, teacher training

\section{AUTEURS}

\section{NATACHA ESPINOSA}

Université Paris Nanterre/CNRS, MoDyCo, UMR 7114, F-92000, France 


\section{SARAH DE VOGÜÉ}

Université Paris Nanterre/CNRS, MoDyCo, UMR 7114, F-92000, France 